\title{
Between astronomy and particle physics: Outreach at the Pierre Auger Observatory
}

\author{
Beatriz García ${ }^{1}$ and Carlos Hojvat ${ }^{2}$ \\ ${ }^{1}$ CONICET, National Technological University FRM and Pierre Auger Observatory, \\ Rodriguez 273, 5500 Mendoza, Argentina \\ email: bgarcia@frm.utn.edu.ar \\ ${ }^{2}$ Enrico Fermi National Laboratory and Pierre Auger Observatory, \\ Box 515, SE-75120 Uppsala, Sweden \\ email: hoefner@astro.uu.se
}

\begin{abstract}
The Pierre Auger Observatory, dedicated to the detection and study of Ultra High Energy Cosmic Rays, has as one of it fundamental missions outreach activities. With this in mind, a specially conceived Visitor Center was included in the design of the Pierre Auger campus in the city of Malargüe, Mendoza, Argentina. One of its main objectives is the contact with the community, through educational courses, talks, conferences and workshops for differen $t$ levels. The permanent exchange between the Pierre Auger scientists and teachers, professionals and society, is important to the impact of the Observatory in the vision by the community of science in general, and scientists in particular. Activities in the outreach area during the last 10 years, resulted in changes to the curricula of study in the schools. Important topics are discussed, like astronomy, light pollution control, and modification of the behavior of the local society. In this presentation we will show how an extensive outreach program, organized by scientists in contact with the community and based on (or near) a research center, can be a factor of social transformation.
\end{abstract}

Keywords. Education in Physics and Astronomy; Outreach: Physics; Outreach: Social Impact,

\section{Introduction}

The Pierre Auger Observatory, in Mendoza, Argentina, is dedicated to the detection and study of Ultra High Energy Cosmic Rays. Outreach is one of its fundamental missions.

Cosmic Rays (CR) are particles that arrive to Earth from the space and hit continuosly our planet from all directions. The objective of the Auger Observatory is the study of these $\mathrm{CR}$ with energies greather than $10^{18} \mathrm{eV}$, with very good angular resolution. The primary cosmic ray particle, interacts with the molecules in the terrestrial atmosphere producing a shower of secondary particles, as shown in Fig. 1 (left).

The purpose of studying these "Messengers from the space", is to find answers to the following questions:

(a) Composition: which kind of particle are CR?

(b) Arrival direction: where do they come from?

(c) Statistics and optical counterpart: Which is the source of such energies?

The Auger Observatory is described as hybrid, because consists of two types of detectors, working at the same time. One measuring the energy deposited in the atmosphere and indicated by fluorescence light and one measuring the distribution of energy at the 
ground (Fig. 1 right). The Observatory covers an area of $3,000 \mathrm{~km}^{2}$ (Abraham et al. 1995).

The International Pierre Auger Observatory Collaboration include Scientists, Engineers, Students and Technicians from 17 countries: Argentina, Australia, Bolivia, Brasil, Czech Republic, Germany, France, Holland, Italy, Mexico, Poland, Portugal, Slovenia, Spain, UK, USA, and Vietnam.

It is important to recognize the difficulties in teaching about elementary particles, because of the abstract concepts involved. Part of the public presentations are diagrams, simulations, and models installed at the Visitor Center of the Observatory.
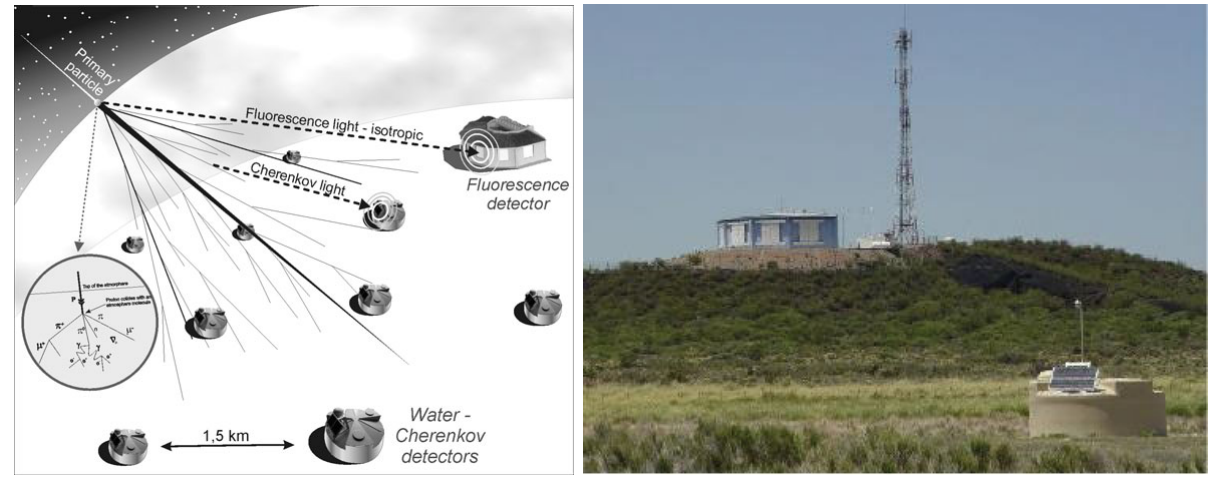

Figure 1. (Lfet) Simulation of extended air shower secondary particles (Silvina Pérez Alvarez, 2009). (Right) The Pierre Auger Observatory is an hybrid Observatory. On the left, one of 4 buildings of Fluorescence optical detectors. To the right is one of 1600 surface detectors.

\section{Visitor Center at Pierre Auger Observatory}

An specially conceived Visitor Center was included in the design of the Pierre Auger campus in the city of Malargüe, Mendoza, Argentina. One of its main objectives is the contact with the local community, through educational courses, talks, conferences and workshops for different levels. Fig. 2 (left) shows the Office building, were the Visitor Center is. Around 7,000 visitors per year, since 2005, assist to the free dayly talks. In Figs. 2 (right) and 3, different groups are visiting the installations.
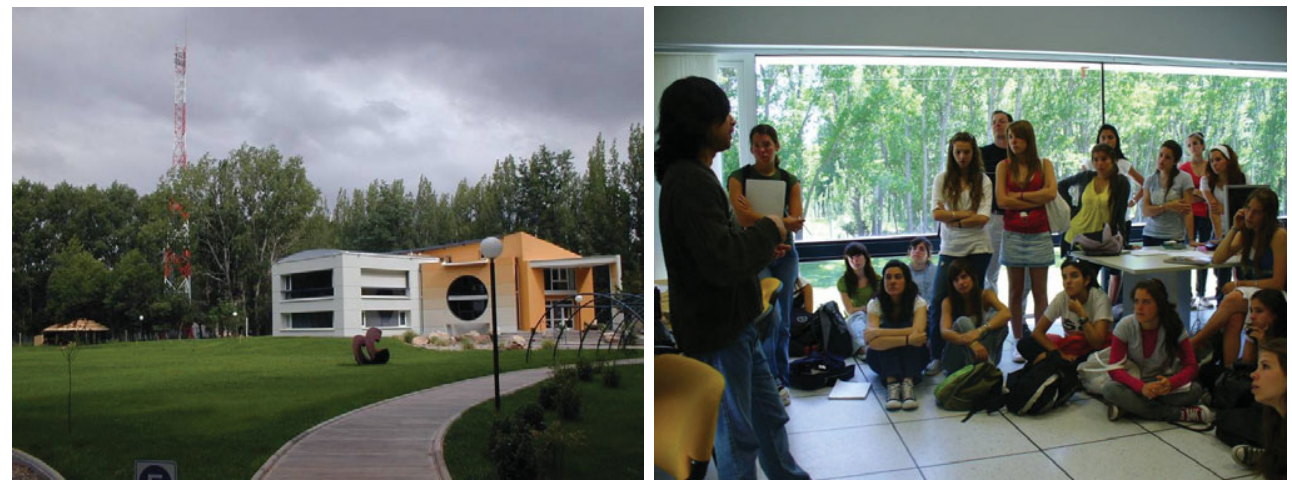

Figure 2. (Left) Headquarters of the Pierre Auger Observatory in Malargüe. (Right) Meeting with high school students at the computer center. 


\section{Social Impact}

The permanent exchange between the Pierre Auger scientists and teachers, professionals and society, is important to analyze the impact of the Observatory in the vision of the community of science in general, and about scientists in particular. Activities in the outreach area during the last 10 years resulted in changes to the curricula of study in the schools. New concepts like astronomy and light pollution control, are now part of the classes; changes on the behaviour of the local community is also clear.

\section{Visits to rural schools}

One special task of the outreach team at the Observatory, is the visit to schools outside the city, in very isolated places. Parts of the workshops specially conceived for this purpose are devoted to give Academic and Technical support; develop material and activities for all the year, in Physics and Astronomy; prepare brochures, pictures, etc.; training Teachers at the schools and at the Observatory, and teaching Methods Course for Science Teachers, in Grades 1-7.

On the other hand, Teachers developed hands-on experiments in response to special problematic situation that appeared during the courses. Topics such us light reflection, gas pressure, motion, are part of the discussion.

\section{Auger Impact Study}

The assessment of the social impact of the Pierre Auger Observatory (PAO) in Malargüe was based on new conceptualizations of organizational management - through Corporate Social Responsibility (CSR) criteria - and on new approaches to the Social indicators "Social Impact" may be said to be the lasting change or group of lasting changes that take place in the society, the economy, the science, the technology and the environment, improving their indicators as the impact of the execution of research, development, and innovating actions that introduce added value to products, services, processes and technologies.

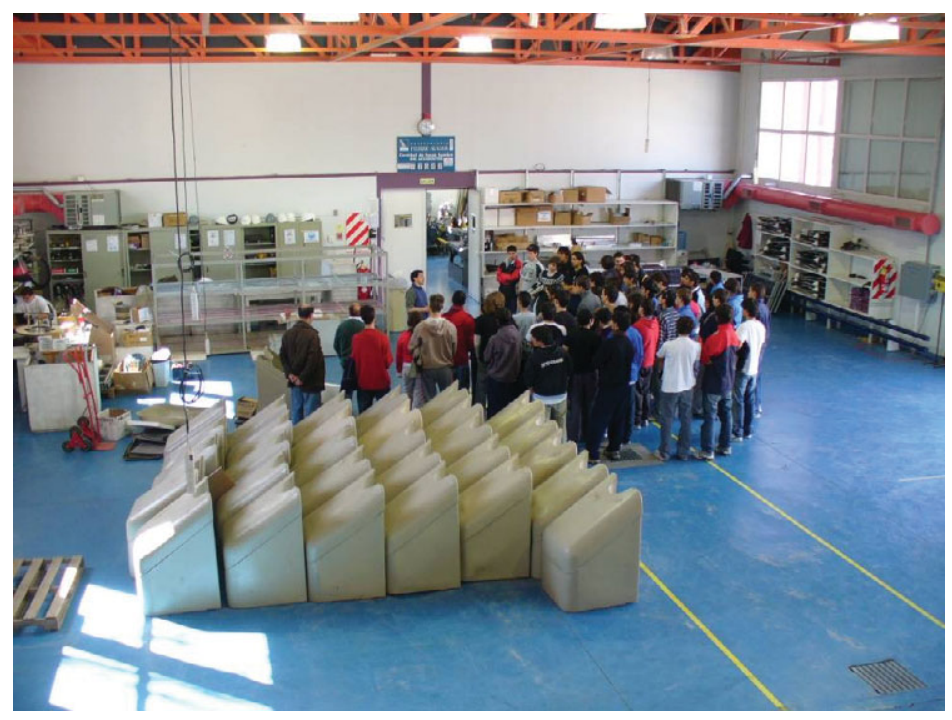

Figure 3. Visit to the assembly building 
According to the above mentioned theoretical frame, it can be said that the arrival of the PAO to Malargüe has fostered the reinforcement of the following set of values:

(a) A growing interest in the sciences.

(b) Promotion of scientific and technological culture.

(c) Increased cultural and environmental awareness.

(d) Effort and training as keys to success in a course of studies or a profession.

(e) Integration

\subsection{PAO and the relationship with the staff}

A permanent staff of 30 employees work at the PAO in Malargüe. These personnel i ncludes physicists, technicians, administrative people. From the impact study was possible to conclude that they: (1) Take pride in working for the PAO, which results in increased identity reinforcement; (2) Feel the need of permanent training and high quality standards at the prospect of getting a job at the PAO.

\subsection{PAO and its relationship with the community and the consumers}

The importance of the scientific institution in small community (Malargüe has about 20,000 inhabitants), help to trigger some processes. We describe only some of them, appeared as part of our impact study:

- There has been a subtle acceleration of the development process.

- Scientific tourism is growing in importance and Malargüe is intended to project an image related to it.

- There has been direct and indirect economic growth of certain sectors (specially due large PAO collaboration meetings, that take place twice a year).

- There has been an increase in direct and indirect employment.

- There have been changes in the attitudes of the local people and the scientists.

\subsection{PAO relationship with the political power}

Some of the joint efforts directed at promoting scientific and cultural initiatives and education are the following:

(a) The promotion of the scholarships to Michigan Technical University, involving the Municipal Council, the Municipal School of English, local schools, the National Technological University and the PAO;

(b) The construction of the Cronin School and the Planetarium (Fig. 4);

(c) The promotion of activities and the guided visits at the observatory, involving the Municipal Council, the Tourism Directorate, the media, tourist companies, individuals, and the PAO.

\section{Activities for International Year of Astronomy 2009}

Part of the outreach activities at PAO during 2009, are in connection to the International Year of Astronomy. We only summarise some of them:

- Dark Skies Awareness (control of the light pollution)and the global activities, Globe at Night and Earth Hour, between others.

- 100 Hours of Astronomy.

- Galileoscope, with the special goal to contruct small telescopes at several schools.

- Eratostenes Project (to measure the dimensions of Earth )

- Aristarco Project (to measure Earth-Moon distance )

- Solar systems in scale, with the city of Malargüe as baseline

- Pendulum of Foucault

- Special shows for the Planetarium 

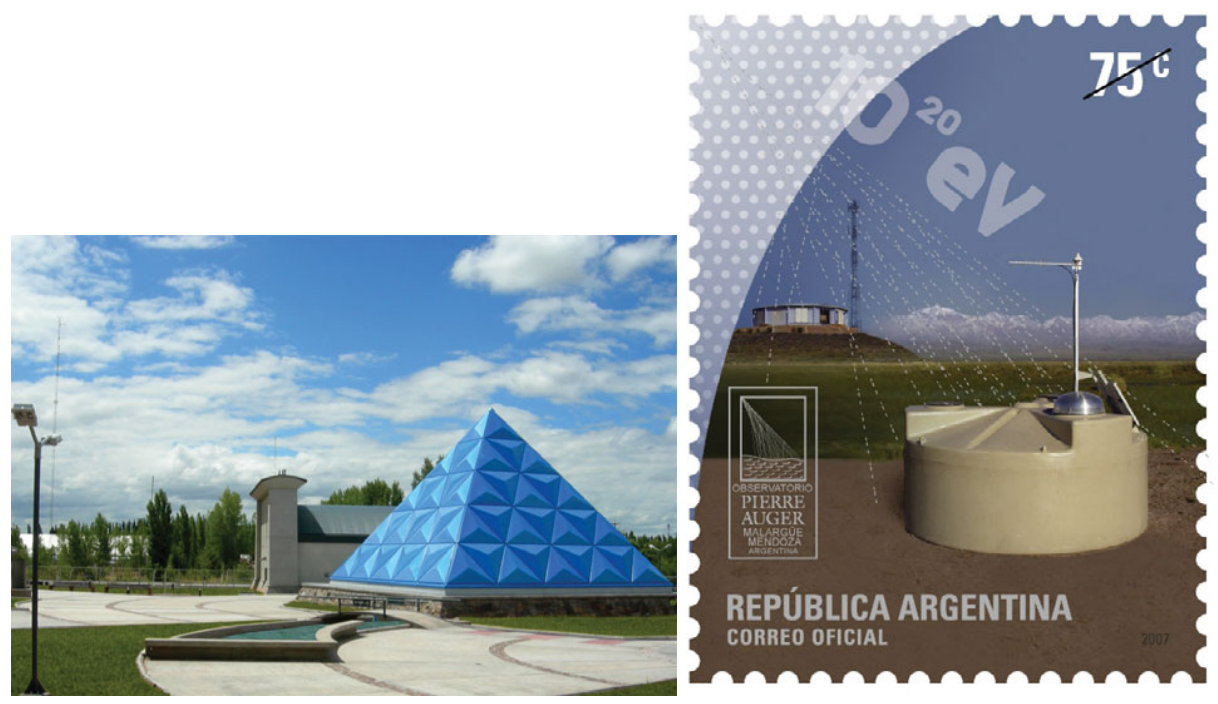

Figure 4. (Left) Planetarium Malargüe. (Right) Post Office stamp, issued in 2006.

\section{Conclusions}

The Pierre Auger Observatory is an institution with a scientific objective that is in agreement to the impacts it makes in Malargüe. The observatory has had a positive effect on its closest sphere of influence, i.e. on the education, tourism, and commercial sectors and -although to a less degree- on the transportation and construction -industries and the public services. According to media experts, even though there are serious outreach efforts, due to the fact that the project is outside the framework of everyday life, the job it is not easy and must to be supported in a long time baseline. Because this is a basic science project with no immediate practical applications, this is probably the main reason why the PAO, despite the outreach efforts, has not been "assimilated" by all the the community.

Finally, as a corollary of the present social impact study, we would like to higlight -in this convulsed and fragmented world of ours- the important contribution of the PAO to the local community as a significant promoter of consensus, integration and openness leading to cultural syncretism. An extensive outreach program, organized by scientists in contact with the community and based on (or near) a research center, can be a factor of social transformation.

Part of the hard work to connect people with the PAO produce some special feature, such us the official post office stamp, issued in 2006 (Fig. 4), the educational videos "Messenger of Space", for children aged 6 to 10, and "An adventure of the mind", for general audiences (for details about this production, see García \& Raschia (2008), the special Google Earth tour, and the Sciences Fair organised by the Observatory staff and the international outreach group.

\section{References}

Abraham, J. et al. 2004, Nucl. Inst. Meth. A, 523, 50

García, B., Raschia, C. 2008, in Innovation in Astronomy Education, J. Pasachoff, R. Ross \& N. Pasachoff (eds), (Cambridge: Cambridge Univ. Press), p. 293 